\section{La recherche de mutations dans I'ADN tumoral circulant aide à prédire la réponse à l'immunothérapie dans le cancer du poumon}

Nicolas Guibert ${ }^{1-3}$, Anne Pradines ${ }^{2,4}$, Gilles Favre ${ }^{2,4}$, Julien Mazières ${ }^{1-3}$

\author{
'Unité d'oncologie thoracique, Hôpital Larrey, CHU Toulouse, \\ Chemin de Pouvourville, 31059 Toulouse Cedex, France. \\ ${ }^{2}$ Inserm, Centre de recherche en cancérologie de Toulouse, \\ CRCT UMR-1037, 31000 Toulouse, France. \\ ${ }^{3}$ Université Paul Sabatier, 31000 Toulouse, France. \\ ${ }^{4}$ Laboratoire de Biologie médicale oncologique, \\ Institut Claudius Regaud, 31059 Toulouse, France. \\ guibert.n@chu-toulouse.fr
}

> Le cancer du poumon «non à petites cellules » (CBNPC) reste la première cause de décès par cancer, même si sa prise en charge a été révolutionnée ces 10 dernières années par l'avènement des thérapies ciblées en présence d'une addiction oncogénique ${ }^{1}$, et plus récemment, par les immunothérapies ciblant les points de contrôle de l'immunité. Le blocage de l'interaction inhibitrice entre PD-Ll (programmed death-ligand 1) exprimé à la surface de la cellule cancéreuse et PD-1 (programmed cell death 1) exprimé à la surface du lymphocyte, en particulier, restaure l'immunité anticancéreuse et permet des réponses prolongées. Cependant, seule une minorité de patients tire un bénéfice durable de ces agents utilisés en monothérapie, qui exposent à un risque d'effet indésirable (auto-immunité). Les biomarqueurs pour identifier ces répondeurs à long terme sont limités: 1) l'analyse de l'expression de PD-Ll dans les tumeurs par immunohistochimie est largement utilisée, mais cette expression varie dans le temps et selon la localistion étudiée dans la tumeur [1] ; 2) la charge mutationnelle tumorale évaluée par le séquençage de l'exome complet permet de prédire une meilleure survie sans progression avec une immunothérapie qu'avec une chimiothérapie, mais ce caractère prédictif n'a pas été confirmé

${ }^{1}$ Dépendance d'un cancer à l'altération d'un gène (ou de quelques gènes) pour maintenir un phénotype cancéreux et assurer la survie des cellules tumorales. en termes de survie globale [2]. Une des limites de cette dernière approche, au-delà de son coût, de sa faisabilité limitée sur tissu [2] et de la lourdeur du traitement bioinformatique des données, réside dans le fait que toutes les mutations ne génèrent pas de néo-épitopes immunogènes et n'ont donc pas la même valeur vis-à-vis de la réponse à l'immunothérapie [3].

$\varepsilon$ n parallèle, des déterminants génomiques de réponse ou de résistance à l'immunothérapie ont été identifiés. II est en particulier démontré que les mutations de KRAS (V-Ki-ras2, Kirsten rat sarcoma viral oncogene homolog), présentes dans $25 \%$ des adénocarcinomes, confèrent à la tumeur une plus grande sensibilité à l'immunothérapie, surtout lorsqu'une mutation de TP53 (tumor protein p53) lui est associée (35 à $57 \%$ de réponse objective à l'immunothérapie contre moins de $20 \%$ dans la population CBNPC globale [4]). À l'inverse, les mutations inactivatrices de STK11 (serine/threonine kinase 11), présentes dans $15 \%$ des adénocarcinomes, en particulier lorsqu'elles sont accompagnées de mutations de KRAS ou de PTEN (phosphatase and tensin homolog), sont associées à de moindres réactions inflammatoires au sein du microenvironnement tumoral et à une résistance à l'immunothérapie (moins de $7,5 \%$ de réponse objective à l'immunothérapie pour les tumeurs avec mutations de KRAS et STK11) [4-6]. Enfin, les réponses à l'immunothérapie des tumeurs avec addiction oncogénique en présence d'une mutation oncogénique d'EGFR (epithelial growth factor receptor) ou d'un réarrangement des gènes ALK (anaplastic lymphoma kinase) ou ROSI (ROS proto-oncogene 1), sont rares [7].

Nous avons fait l'hypothèse qu'un séquençage ciblé de l'ADN des cellules tumorales, limité à ces anomalies, pourrait prédire la réponse à l'immunothérapie. À défaut d'une quantité résiduelle de tissu disponible avec des biopsies de petite taille, nous avons analysé I'ADN tumoral circulant (ADNtc), correspondant à I'ADN des cellules tumorales présent dans la circulation sanguine. Cette analyse peu invasive, qui peut être répétée au cours de l'évolution du cancer sous traitement, permet de prendre en compte l'hétérogénéité du profil moléculaire de la tumeur dans le temps et dans l'espace. Nous avons également étudié la corrélation entre les variations précoces (1 mois) du taux d'ADNtc et la réponse au traitement.

Notre étude [8] a porté sur une cohorte de 97 patients atteints d'un CBNPC de stade avancé (stade IIIB/IV) et débutant une immunothérapie en seconde ligne après un échec de la chimiothérapie (IMMUNOPREDICT, NCT02827344). La recherche des mutations de I'ADN plasmatique a été réalisée à partir d'un échantillon de sang par une technique de séquençage à haut débit (Inivata, InVision First ${ }^{\circledR}$ - Lung technology, Morrisville, 


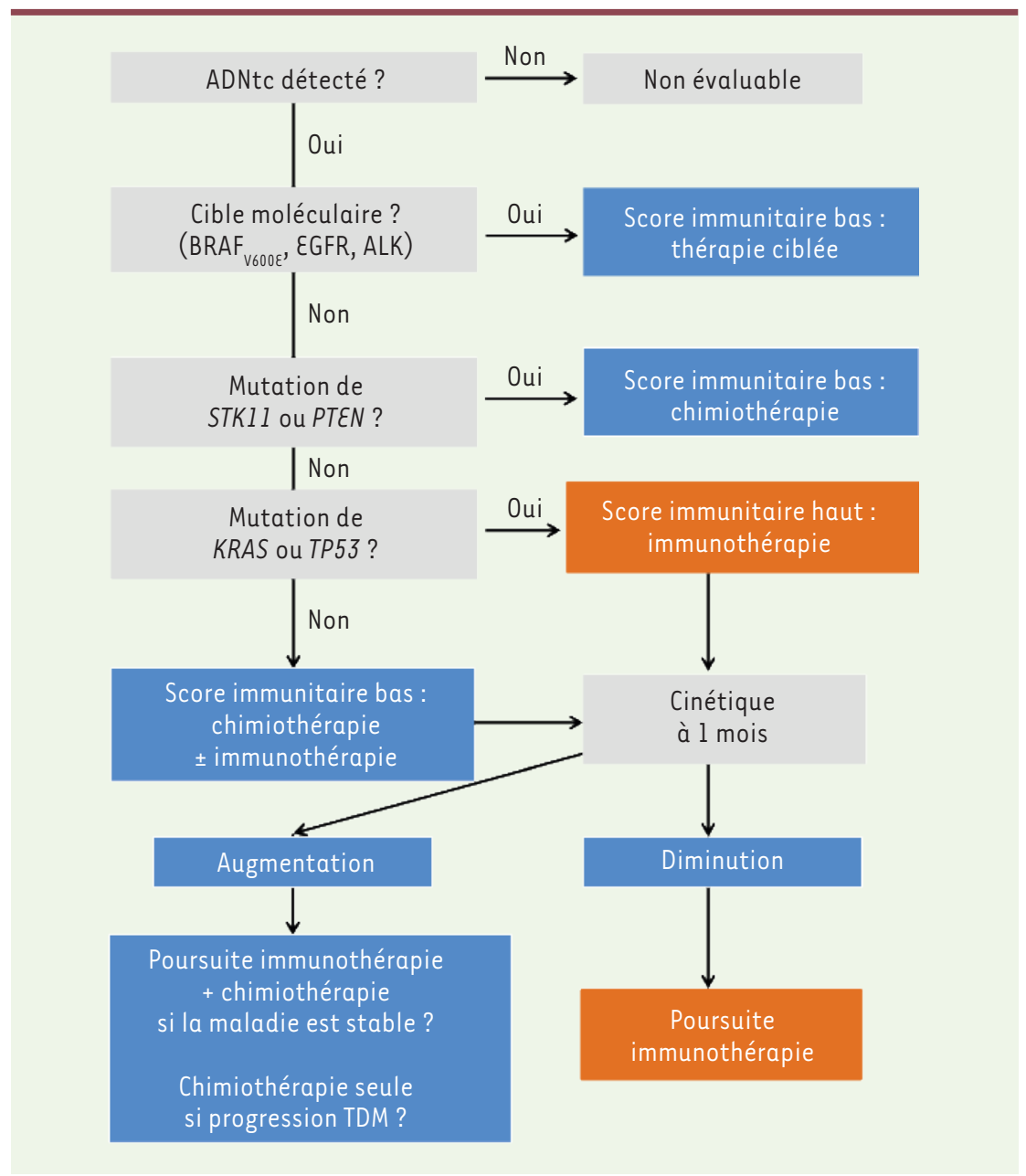

Figure 1. Proposition d'utilisation de l'ADN tumoral circulant (ADNtc) pour guider l'immunothérapie. Si un driver oncogénique est détecté, une thérapie ciblée, ou une chimiothérapie si les possibilités de thérapie ciblée ont été épuisées, doit être préférée. La présence d’une mutation de PTEN ou de STKII doit faire reconsidérer la pertinence d'une immunothérapie, alors qu'une mutation par transversion de KRAS ou de TP53 est prédictive de bons résultats. Un « score immunitaire haut », défini par l'absence de driver oncogénique et de mutations de PTEN ou de STK11, mais la présence de mutations par transversion de KRAS ou de TP53, prédit un résultat favorable de l'immunothérapie. Une diminution de la concentration de l'ADNtc à un mois est fortement prédictive d'une réponse durable ( $74 \%$ de patients en réponse à 6 mois) et doit motiver la poursuite de l'immunothérapie, alors qu'une augmentation initiale indique une probable progression tumorale ( $84 \%$ de patients avec progression de la tumeur dans les 6 mois) et incite à modifier le traitement (notamment si l'imagerie par tomodensitométrie (TDM) indique également une progression de la maladie) ou peut-être à y associer une chimiothérapie si l'imagerie indique une stabilité de la maladie.

NC, États-Unis), sur un panel de 36 gènes d'intérêt. L'ADNtc a été détecté dans $78 \%$ de ces prélèvements $(67 / 86)$. Aucun des patients porteurs d'une addiction oncogénique (hors mutations de KRAS) n'a répondu à l'immunothérapie (5 patients avec mutation de l'EGFR,
1 patient avec réarrangement de $A L K)$. La présence d'une mutation de PTEN ou de STKIl était associée à une moindre réponse (rapport des risques instantanés ou hazard ratio : 8,9 pour PTEN, et 4,7 pour STK11). À l'inverse, les mutations par transversion de KRAS ou TP53 étaient associées à de meilleures survies sans progression (hazard ratio: 0,36 pour TP53 et 0,46 pour KRAS).

Nous proposons un arbre décisionnel tenant compte du profil moléculaire de I'ADNtc (Figure 1). Le «score immunitaire haut » $(\mathrm{SIH})$ est défini par l'absence d'une altération des gènes drivers $^{2}$ ( $\varepsilon G F R, R O S 1$, ALK et $B R A F_{\mathrm{V} 600 \varepsilon}$ ) et de mutations de STKIl ou PTEN, et la présence de mutations par transversion de KRAS ou TP53. Le SIH était associé à une médiane de survie sans progression de 14 mois et un taux de réponse à 6 mois de $76 \%$. Le score immunitaire est considéré comme «bas » (SIB) en présence d'une altération d'un gène driver (hors KRAS), de PTEN ou de STK11, et en l'absence de mutations par transversion de KRAS ou TP53. Le SIB était associé à une médiane de survie sans progression de 2 mois, et un taux de réponse à 6 mois de $33 \%$ (Figure 1).

Les patients dont la concentration plasmatique de l'ADNtc augmente dans le premier mois ont une moins bonne réponse à l'immunothérapie (médiane de survie sans progression de 2 mois, taux de réponse à 6 mois de $16 \%$ ) que ceux dont la concentration diminue (médiane de survie sans progression de 10 mois). La cinétique est d'autant plus discriminante que la variation est grande : seulement $11 \%$ et $6 \%$ de taux de réponse à 6 mois après une augmentation de 30 ou $50 \%$, respectivement, de la concentration de l'ADNtc.

L'identification, dans le plasma, de manière plus ciblée qu'avec la charge mutationnelle tumorale, des mutations associées à une résistance (mutations driver, STK11, PTEN) [5-7] ou, au contraire, à une plus grande sensibilité à l'immunothérapie (mutations par transversion de KRAS ou de TP53) $[4,9]$ permet donc de sélectionner les patients répondeurs à l'immunothérapie, avec des médianes de survie sans progression

\footnotetext{
${ }^{2}$ Gène dont l'altération (mutation, réarrangement chromosomique entraînant une fusion avec un autre gène, etc.) entraîne une addiction oncogénique.
} 
de 2 mois pour les patients avec SIB, et de 14 mois pour les patients avec $\mathrm{SIH}$. De la même manière, la cinétique précoce de I'ADN tumoral circulant est un assez bon indicateur de la réponse à 6 mois, même s'il est imparfait (e.g., immunothérapie efficace à 6 mois chez $11 \%$ des patients dont la concentration de l'ADNtc a augmenté de plus de $30 \%$ à un mois) et ne doit probablement être envisagé qu'en complément de l'imagerie, qui est parfois d'interprétation délicate (e.g., réponse lente à l'immunothérapie, ou vraie progression de la tumeur considérée à tort comme une pseudo-progression).

Une limite de cette étude est son caractère rétrospectif et l'absence de groupe témoin (patients traités par chimiothérapie par exemple), qui empêche de différencier de manière formelle un effet prédictif sur la réponse à l'immunothérapie d'un effet pronostique. L'arbre décisionnel proposé dans la Figure 1, présenté à titre indicatif, ne serait utilisable qu'en cas de validation des résultats dans une étude prospective.

$\varepsilon$ n conclusion, le séquençage à haut débit d'un nombre limité de gènes dans I'ADNtc, répété au cours du temps, pourrait constituer un outil complémentaire pour prédire et suivre la réponse à l'immunothérapie dans le cancer du poumon. $\diamond$

Finding mutations of interest in circulating tumor DNA helps predict immunotherapy response in lung cancer

\section{LIENS D'INTÉR}

Les auteurs déclarent n'avoir aucun lien d'intérêt concernant les données publiées dans cet article.

\section{RéFÉRENCES}

1. Ilie M, Long-Mira $\varepsilon$, Bence C, et al. Comparative study of the PD-LI status between surgically resected specimens and matched biopsies of NSCLC patients reveal major discordances: a potential issue for antiPD-L1 therapeutic strategies. Ann Oncol 2016; 27 : 147-53.

2. Hellmann MD, Paz-Ares L, Bernabe Caro R, et al. Nivolumab plus Ipilimumab in advanced non-smallcell lung cancer. $N$ Engl J Med 2019 ; 381 : 2020-31.
3. Chen DS, Mellman I. Elements of cancer immunity and the cancer-immune set point. Nature 2017 ; 541 321-30.

4. Skoulidis F, Goldberg ME, Greenawalt DM, et al. STK11/ $\angle K B 1$ mutations and PD- 1 inhibitor resistance in KRAS-mutant lung adenocarcinoma. Cancer Discov $2018 ; 8: 822-35$.

5. Peng W, Chen JQ, Liu C, et al. Loss of PTEN promotes resistance to T cell-mediated immunotherapy. Cancer Discov 2016 ; $6: 202-16$.

6. Koyama S, Akbay EA, Li Yy, et al. STK11/LKB1 deficiency promotes neutrophil recruitment and proinflammatory cytokine production to suppress T-cell activity in the lung tumor microenvironment. Cancer Res 2016; 76 : 999-1008.

7. Mazieres J, Drilon A, Lusque A, et al. Immune checkpoint inhibitors for patients with advanced lung cancer and oncogenic driver alterations: results from the Immunotarget registry. Ann Oncol 2019; 30 1321-8.

8. Guibert N, Jones G, Beeler JF, et al. Targeted sequencing of plasma cell-free DNA to predict response to PDI inhibitors in advanced non-small cell lung cancer. Lung Cancer Amst Neth 2019 ; 137 : 1-6.

9. Rizvi H, Sanchez-Vega F, La K, et al. Molecular determinants of response to anti-programmed cell death (PD)-l and anti-programmed death-ligand l (PD-LI) blockade in patients with non-small-cell lung cancer profiled with targeted next-generation sequencing. J Clin Oncol 2018; 36 : 633-41.

P ossédées du Malin au Moyen-Âge, les sorcières hystériques sont vouées au bûcher. Enfermées au xvıI siècle, maltraitées, elles rejoignent la Cour des Miracles de l'Hospice de la Vieillesse-Femmes à la Salpêtrière... Jusqu'à ce que le Dr JeanMartin Charcot (1825-1893) mène le combat qui transforme l'ancien hospice en hôpital : l'École de la Salpêtrière de

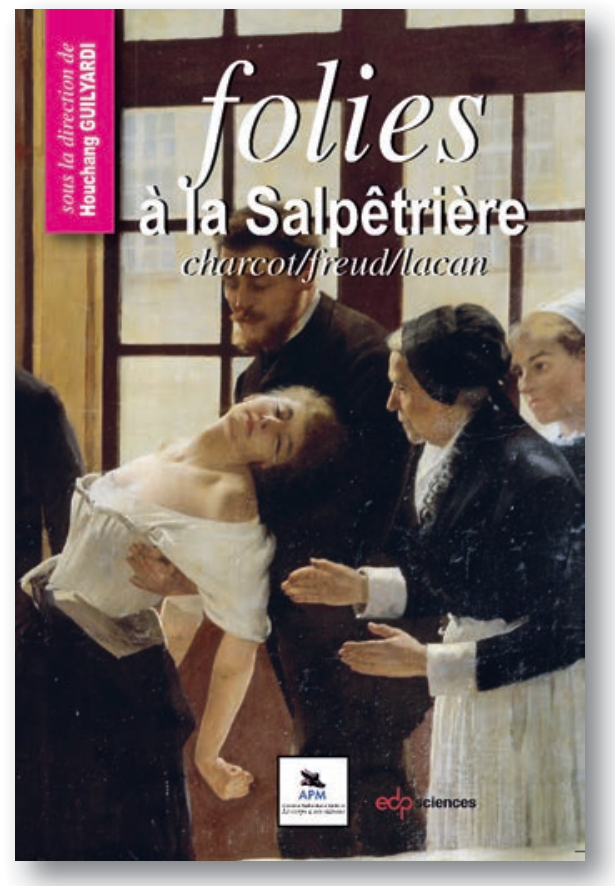

ISBN : $978-2-7598-1268-4$
240 pages Paris est née, qui devient lieu de recherche, d'enseignement et de soins, de renommée internationale.

Jean Martin Charcot n'a pas bonne presse, et pourtant... Hystérie et folie traversent les siècles, prenant les formes de «l'air du temps ».

De l'utérus migrateur d'Hippocrate aux recherches neurologiques de Charcot. Du désir inconscient avec Freud à la jouissance du parlêtre chez Lacan... C'est à cette traversée historique et conceptuelle que nous convie cet ouvrage.

À retourner à EDP Sciences, 17 avenue du Hoggar, 91944 Les Ulis Cedex, France Tél. : 0149856069 - Fax : 0149850345 - $\varepsilon$-mail : francois.flori@edpsciences.org

NOM :

Adresse :

Code postal : Prénom :

Pays :

Fonction:

Je souhaite recevoir

Folies à la Salpêtrière : $20 €+3 €$ de port $=23 € \pi T$

en ................... exemplaire, soit un total de ............................................ $€$

$\square$ Par chèque, à l'ordre de EDP Sciences

$\square$ Par carte bancaire : $\quad \square$ Visa $\square$ Eurocard/Mastercard

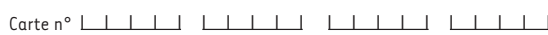

Date d'expiration : $\sqcup \sqcup \sqcup \sqcup N^{\circ}$ de contrôle au dos de la carte : $\longleftarrow \perp \sqcup \sqcup$ Signature : 